\title{
Relation of Memory and Linguistic Fields and ICT Tools for Memory and Language Comprehension
}

\author{
https://doi.org/10.3991/ijes.v5i1.6275 \\ Aglaia Tourimpampa \\ National Center for Scientific Research "Demokritos", Agia Paraskevi, Attica, Greece \\ lidatou@gmail.com \\ Athanasios Drigas \\ National Center for Scientific Research "Demokritos", Agia Paraskevi, Attica, Greece \\ dr@iit.demokritos.gr \\ Alexandra Economou \\ University of Athens, Athens, Greece \\ aoikono@psych.uoa.gr
}

\begin{abstract}
The purpose of this paper is to investigate the impact of human memory in language comprehension and specifically to examine the relationship between the function of working memory and the fields of linguistic analysis which are phonology, morphology, syntax, semantics and pragmatics. Working memory -as one of the most important cognitive skills of human braincomprises a system with specific functions with which the information from the external environment are being transferred, encoded, stored and retrieved. The present study is an attempt to explore which specific function of working memory is activated in order to decode each linguistic field of analysis. Furthermore, it features the current scientific achievements on the ICTs processes and tools, which exploit the cognitive skill of memory and they are applied in the assessment of difficulties in text comprehension, intervention and training of the language comprehension ability.
\end{abstract}

Keywords-Working Memory, linguistic fields, ICT Tools, memory assessment, memory training, language comprehension

\section{$1 \quad$ Introduction}

Memory is the process in which information is encoded, stored, and retrieved. Encoding allows information from the outside world to be sensed in the form of chemical and physical stimuli. In the first stage the information must be changed so that it may be put into the encoding process. Storage is the second memory stage or process. This entails that information is maintained over short periods of time. Finally the third process is the retrieval of information that has been stored. Such information must be located and returned to the consciousness. Some retrieval attempts may be effortless 
due to the type of information, and other attempts to remember stored information may be more demanding for various reasons.[1]

Memory as a fully featured cognitive system has individual storage systems which are Sensory Memory, Short Term memory and Long Term Memory. These three are not separate, mutually exclusive entities found in separate brain centres. . They differ in terms of the functions they perform and their capacity for retaining information for a specific period of time i.e. for how long can they keep the information stored. Specifically, Sensory memory refers to the initial, momentary recording of information in our sensory systems. Short Term Memory refers to the ability to hold information in mind for a brief period of time. As conceptions of Short-Term Memory expanded to include more than just the brief storage of information, psychologists created new terminology. The term Working Memory is now commonly used to refer to a broader system that both stores information briefly and allows manipulation and use of the stored information. This system is higher in functioning than Sensory Memory, as it stores information in terms of meaning and not just simple sensory stimulation. Sensory information is meaningless and therefore discarded. If it is sent to the ShortTerm Memory then a meaning is added to it. Short Term Memory retains information for 15 to 25 seconds, unless it is moved into the Long Term Memory. The component of Working Memory is Central Executive which coordinates the material to focus on during reasoning and decision making and two sub components; the Visuospatial Sketch Pad which concentrates upon visual and spatial information and Phonological Loop that manipulates material related to speech, words and numbers[2].

In order to investigate the impact of the human memory in text comprehension we have to study the special relationships between every component of memory and the linguistics fields of analysis. More specifically, we attempt to investigate which part of memory is activated in order to decode every field of linguistic analysis aiming the text comprehension. The cognitive process of language understanding comprises the linguistic fields of phonetics, morphology, syntax, semantics and pragmatics. Phonetics is the linguistic field that studies the production of speech and the perception of the produced sounds by human brain. Morphology studies the formation of words from smaller units. Syntax is the linguistic field that investigates all the rules that control the formation of the sentences of words. Semantics is the branch of linguistics that studies the meaning of the language and Pragmatics studies the meaning of the context and how language is used to communicate rather than how it is internally structured. In this paper through the presentation of the related studies, there is an attempt to examine which component of memory is activated more in relation with the linguistic fields in the process of text or speech comprehension. Namely, we illustrate findings of experiments which examine this relation. The categorization of the related studies in this article is based on the linguistic fields.

Moreover, this paper offers a descriptive account of the role of ICTs in the assessment of the specific relationship between components of memory and text or speech comprehension. 


\section{$2 \quad$ Memory and Linguistic Fields}

\subsection{Memory and Phonetics}

The focus on current research in the field of memory is on the investigation of the phonological loop, the component of working memory that has an impact on reading comprehension and seems to have an internal relation with the linguistic field of phonetics. Susan E. Gathercole and Alan Baddeley [3] attempt to evaluate the role of phonological Short-term Memory in the development of vocabulary in children. Vocabulary acquisition by 104 children between the ages of 4 and 5 were tested and retested 1 year later. On both occasions, children were asked to repeat back nonwords varying in length and complexity, while nonverbal intelligence and reading were assessed using standard tests. The phonological memory score was highly correlated with vocabulary at both age $4(r=.525)$ and age $5(r=.572)$. Phonological memory at age 4 also demonstrated a significant amount of variance in vocabulary score at age 5 , over and above that accounted for by the vocabulary score the previous year.

Erika Hoff, Cynthia Core and Kelly Bridges [4] in their experiment, that concerned children younger than two years old, made an interesting approach connecting individual differences in phonological memory to the non word repetition task (NWT) and to vocabulary development. There were two studies in which NWR accuracy, real word repetition accuracy and productive vocabulary were assessed. In both studies, NWR accuracy was notably related to vocabulary and, furthermore, because of this accuracy there was a variance in vocabulary when real word repetition accuracy was held constant. The findings consolidate NWR as a valid measure of phonological memory in very young children, and provide suitable conditions for further studies of the role of phonological memory in early word learning.

Alan D. Baddeley, Neil Tomson and Mary Buchanan [5] examine the impact of word length to be recalled in order to study the function of immediate memory span. Results indicated that memory span depends on the word length. Moreover, When number of syllables and number of phonemes are held constant, words of short temporal extent are better remembered than words of long extent. Based on the number of words which the subject can read in approximately $2 \mathrm{sec}$, there can be a prediction of the span. Lastly, when articulation is held back by requiring the subject to articulate an irrelevant sound, the word length effect disappears with visual presentation, but remains when the presentation is auditory.

\subsection{Memory and Syntax}

In this work it is significant to point out also the correlation between the role of syntactic processes and the function of working memory in text comprehension. Walter Kintsch, David Welsch, Franz Schmalhofer and

The research suggestion of Maryellen C. MacDonald, Marcel Adam Just and Patricia A. Carpenter [6] underlines the effect of Working Memory in syntactic parsing and the process of syntactic disambiguation. This hypothesis was based on four experiments that measured reading times for two texts that contain syntactic ambiguity. The 
results of the experiments showed that both interpretations of the disambiguation process were noted but there was a difference on the duration of their maintenance due to the working memory capacity. The process of word-by -word reading time needed extra procedures for disambiguation. Consistent to the above, readers with larger working-memory capacities maintain both representations for some period of time (several words), whereas readers with smaller working-memory capacities managed to maintain only the more likely representation.

The experiment of Evelina Fedorenko, Edward Gibson, Douglas Rohde [7] gives prominence to the role of working memory in sentence comprehension. Participants read sentences of different syntactic structure (containing subject- and objectextracted relative clauses) while remembering one or three nouns (similar to or dissimilar from the sentence-nouns). Results demonstrated a correlation between syntactic complexity and similarity between the memory-nouns and the sentence-nouns in the three memory-nouns conditions. Furthermore, the similarity between the memorynouns and the sentence-nouns influenced the more complex object-extracted relative clauses to a further point than the less complex subject-extracted relative clauses.

Richard L. Lewis and Shravan Vasishth and Julie Van Dyke [8] motivated by the assumption that sentence comprehension is connected to the function of working memory capacity, in order to be computed different linguistic relations within the sentence, they propose a theoretical structure for the working memory system that comprises several principles of memory: a sharply limited attentional focus, rapid retrieval of item (but not order) information subject to interference from similar items, and activation decay (forgetting over time). A computational model incorporating these principles demonstrates an explanation of the functional capacities and limitations of human processing, as well as accounts of reading times.

\subsection{Memory and Semantics}

Semantics is the linguistic field highly related to Semantic Buffer, one of the Working Memory's components and it has a main role in text comprehension. John B. Black [9] and Hyman Bern in their experiment tried to investigate how coherent causality between sentences from stories has an effect in remembering the cue of these sentences. In a second experiment, subjects free recalled the same stories. Again, memory was activated better when the sentences when they were part of a causally related pair. Also subjects were more likely to combine two sentences into one during recall when they were causally related.

Another element that rises from the hypothesis expressed by Gail McKoon [10] is that memory as far as concerns text comprehension reinforced when text is structured hierarchically and the information is presented on the basis of its importance. Subjects read short texts and then they were asked to check if sentences of the text were true or false. Sentences that concerned topic information were verified faster that others that contained details information. These results were made when testing immediately followed the text. But when testing was delayed, the effects were different. Subjects did not verified the same sentences as far as concerns their importance. Therefore, the investigation jumps to the conclusion that the hierarchical order of information from 
the most important to the least important in the text, effects memory in the process of text comprehension.

Also, Maria N. K. Schwartz and August Flammer [11] indicated in their experiments the effect of a thematic title in the recall of a structured text. Moreover, Experiment 2 outlines that even prolonged reading time reinforces the role of the thematic title in remembering even a structured text without a regular structure. Furthermore, the two experiments demonstrated the comprehensibility ratings to be affected by the text structure and the reading time, but not by the title. Another semantic procedure that is related with the activation of Working Memory's Semantic Buffer is inference.

Gail McKoon and Roger Ratcliff [12] examined inference processes involved in anaphoric reference in three experiments. In the first two experiments the subject read a paragraph sentence by sentence and then, a single test word from the first sentence of the paragraph is given for recognition. The subject recognized immediately the test word when it was a referent of an anaphor mentioned in the last sentence of the paragraph and when the test word was in the same sentence as the referent of the anaphor in the last sentence. This shows that an anaphor activates both its referent and concepts in the same proposition as its referent. The third experiment showed that the referent and a concept in the same sentence as the anaphor are activated by the longterm memory in text comprehension. These results are discussed in terms of a simple three-process model of anaphoric inference.

Akira Miyake, Marcel Adam Just and Patricia A. Carpenter [13] in their paper study the impact of Working Memory 's capacity in the process lexical ambiguity explanation during text comprehension. Experiments showed that the more strong working Memory capacity is the more easy is for the reader to interpret ambiguous words. In the first experiment subjects read sentences with neutral contexts which were followed by a homograph that disambiguated much later. Results showed that high span readers faced little difficulty in encountering lexical ambiguity while low span readers did not manage to interpret all meanings of disambiguation.

\subsection{Memory and Pragmatics}

In this part of the paper it is given a comprehensive account of the correlation between Episodic Buffer and Pragmatics. John B. Black and Gordon H. Bower [14] in their paper suggest that as narrative stories chunk into episodes, memory representations of these stories can cluster into episodes too. In order to confirm this hypothesis of the chunking idea, researchers made an experiment showing that the recall of the certain episodes depends on the length of that episode. Consistent to this hypothesis, the recall of the episode was reinforced by adding more actions to it, while recall of other episodes was not affected.

Valerie Abbott, John B. Black and Edward Smith[15] investigated how common events are represented in memory and the inferences people make in order to understand when they read the descriptions of these events. Readers had a tendency in making inferences of items at the level of abstraction when they read a sentence that contained an abstract concept. Results indicated that scripted events are represented in memory as hierarchically and temporally organized information packets. 
An interesting approach related to the effect of cognitive structures and content on memory and text comprehension, has been proposed by Perry W. Thorndyke [16]. A class of simple narrative stories described by a plot of structures was used in two experiments. The examination of readers' story memory function was based on the assumption that memory constructs an hierarchical organizational framework of the stories that represents the abstract structural components of the plot. Comprehensibility and recall turned out to be correlated to the amount of inherent plot structure in the story, independent of passage content. Recall probability of individual facts from passages depended on the structural centrality of the facts, while subjects' recall of the story depended on how well the story was organized. Moreover, story summarizations from memory tended to emphasize general structural characteristics rather than specific content.

\section{$3 \quad$ Processes and ICT Tools for Memory and Language Comprehension}

In this work it is also attempted the presentation of the current research effort that has been made on the field of ICTs and the assessment of memory deficit and memory training. This paper reports some indicative studies on the ICTs that focus on memory training for text comprehension. Mina C. Johnson Glenberg [17] displays the web-based tool application called 3D-Readers which applies meta cognitive reading strategies in poor reading comprehenders and focuses on the verbal and visual strategies connected to the Memory Visuospatial sketchpad. The results showed that the incorporation of verbal and visual strategies were very effective and reinforced reading comprehension and poorer comprehenders reoriented their text comprehension process.

Jerker Rönnberg, Mary Rudner, Catharina Foo and Thomas Lunner [18]describe a working memory system that enhances language comprehension (ELU, Rönnberg). The activation of cognitive functions for this aim is demonstrated in this work.Connected to speech understanding, the system based on (1) the quality of phonological representations in long-term memory, (2) phonologically mediated lexical access speed, and (3) explicit, storage, and processing resources. If there is a discrepancy between phonological information extracted from the speech signal and the phonological information exemplified in long-term memory, the system is assumed to produce a discrepancy signal that implores explicit processing resources. In the present study, four aspects of the model are examined: the language generality assumption; the discrepancy assumption; chronological age; and the episodic buffer function of rapid, automatic multimodal binding of phonology (RAMBPHO). Language generality hypothesis is assessed as far as concerns sign language and speech, and the discrepancy expectation, regarding signal processing in hearing aids. Furthermore, the effects of chronological age and the implications of RAMBPHO are evaluated.

Tracy P. Alloway, Susan E. Gathercole, Hannah Kirkwood \&Julian Elliott [19] in their study evaluate the standardized computerized tool for assessing working memory: the Automated Working Memory Assessment (AWMA). This tool gives the 
chance to users to assess memory deficits. Results have indicated that working memory capacity in children with memory deficit is stable over a school year.

Janet Marie Wasowicz and Art Carl Maerlender [20] have created an assessment system for estimating one or more phonological awareness, phonological processing and reading skills of an individual to reveal phonological awareness, phonological processing and reading skill deficiencies in the individual in order to limit down the possibility of enhancing a reading deficiency and to reduce existing reading impairments.

Sergio Della Sala Colin Gray, Alan Baddeley, Nadia Allamano and Lindsey Wilson [21] evaluate the indication that non-verbal short-term memory has distinct visual and spatial/sequential components. For that purpose, they demonstrate The Visual Patterns Test (VPT) which was designed to measure short-term visual memory largely independent of its spatio-sequential component. Also, studies on the Corsi Blocks Test ,related to the VPT indicate that visual and sequential abilities are not related. Furthermore, in an intervention experiment, the VPT and the Corsi tests were found to show a double dissociation pattern of intervention from visual and spatio-sequential tasks. As a conclusion, non-verbal short-term memory can indeed be seen as specific visual and spatio-sequential components.

Athanasios Drigas, Georgia Kokkalia and Miltiadis D. Lytras [22] regarding the impact of memory deficits in language learning, they present a review of the most representative ICT tools for assessment and intervention of memory impairments in kindergarten.

Tracy Packiam Alloway, Susan Elizabeth Gathercole, Hannah Kirkwood and Julian Elliott [23] establish the function of Working Memory Rating Scale (WMRS), which correlates with behavioural difficulties of children with poor working memory. The evaluation of the scale as far as concerns assessment processes of memory deficits was remarkable. In general, WMRS gives teachers the advantage of early detection of children with memory difficulties.

Elena Aristodemou, Tatjana Taraszow, Yiannis Laouris ,Timotheos Papadopoulos and Pantelis Makris [24] raise the importance of the critical issue of reading difficulties and illustrate the fact that this deficit can be related to broader cognitive difficulties or dyslexia. In addition, they note that the more prompt is the assessment and intervention of the problem the more effective is its' confrontation. For this reason, they demonstrate in their paper the development of an Internet based applications battery which has an assessment function for children's cognitive disabilities and constitutes a tool for assisting teacher to apply the right repairing programs. This study evaluated the capability of the new computerized cognitive battery of tests to predict reading performance. The MAPS (Mental Attributes Profiling System) battery consists of eight major domains of language-independent tests that have been frequently linked to reading development and/or disability: (1) short-term visual memory, (2) short-term auditory memory, (3) auditory discrimination, (4) visual discrimination, (5) lateral awareness, (6) categorization, (7) sequential processing, and (8) navigational ability. A set of Rapid Naming of pictures and letters and word reading tests (Word Identification and Word Attack) of students attending Grade 2, 4 and 6 was used as the dependent variable of MAPS in predicting reading performance. 
Results illustrated five elements as the most capable of predicting reading performance; these were lateralization, auditory memory, categorization, sequencing and auditory discrimination.

Georgia K. Kokkalia, and Athanasios S. Drigas [25] offer in their paper a considerable description of the Automated Working Memory Assessment (AWMA), a computerized measure for educational professionals to detect memory problems in individuals aged 4-22 years old. General speaking, AWMA is a computer-based assessment that offers three measures each of verbal short-term memory, visuo-spatial short-term memory, verbal working memory, and visuo-spatial working memory. Specifically, AWMA- short form (AWMAS) is used for detecting learners who are suspected to have memory deficits, but the specific area of their difficulties is not known and AWMA- long form (AWMA-L) which is suitable for confirmation of working memory problems for learners identified as having working memory problems in the classroom. According to the developers, AWMA includes three levels of assessment. The test includes 12 subtests of verbal Short Term Memory (Digit Recall, Word Recall and Nonword Recall), visuo-spatial Short Term Memory (Dot Matrix, Block Recall and Mazes Memory), verbal Working Memory (Backward Digit Recall, Listening Recall and Counting Recall), and visuo-spatial Working Memory (Mr X, Spatial Span and Odd-One-Out). Lastly, the AWMA is the first standardized tool for non-specialist assessors such as classroom teachers to use so as to become aware of the working memory problems of their pupils quickly and effectively. The authors of this paper also point out Cognitive Profiling System (CoPs), a computerized psychometric assessment system that identifies the cognitive strengths and difficulties for kindergarten and primary school children. CoPS is consisted of eight tests in the form of game, included phonological awareness, auditory discrimination, short term visual memory and auditory verbal memory. The total assessment time lasts twenty minutes and is used across the UK, Scotland and British schools around the world. According to the developers, the students are tested also, in sequential and associative memory, in auditory and colour discrimination and in phonological awareness providing a picture of the child's level of development in the relevant areas at the time of school entry. Another remarkable software tool developed for students with memory difficulties is APLo on-line system. It is proposed for the assessment of children's oral speech and language abilities. Specifically, APLo diagnoses articulation, phonological, fluency, and language deficits.According to the researchers, the teacher selects suitable activities in a digital environment that can be used in the preschool setting or at the first school age to enhance phonology activities aiming to enhance phonological awareness, communication skills and accomplish the best learning results. Furthermore, another software tool developed for the same purpose as the above is edu- Sensus. It is an intervention tool that designed for speech therapists and covers a wide range of topics in the use of ICT tools in supporting the development of children who face dyslexia or other problems such as memory and attention deficits. Authors give also great attention to Memory Booster, Booster is a computer game that teaches memory strategies, over a period of six to eight weeks and reinforces the use of rehearsal, visual imagery, creating stories and making groups. The tool, according to many studies seems to have a wide effectiveness in working memory tasks assessing 
the phonological loop and central executive components of working memory. And last but not least, authors report the Working Memory Test Battery standardized for children (WMTBC). The test includes four measures of verbal short-term memory: digit recall, word recall, non-word recall, and word list matching recall. All four measures involve verbal presentation of memory items. Individual must be capable to answer immediately what recalled from three first tests. Word list matching demands the capacity of the child to decide whether two spoken sequences are identical or not, as it places minimal demands on phonological and articulator production skills. The WMTB-C also includes three measures of verbal working memory (listening recall, counting recall, and backwards digit recall), scores on which are used to give a mixed working memory score. Finally, the WMTBC includes three measures of visuospatial short-term memory (matrices, mazes memory, and block recall).

\section{Discussion}

The purpose of this paper was to draw the scientific interest in the investigation of the impact of memory functions in text comprehension and in language acquisition in general. As it was mentioned above, working memory is an integrated cognitive system and comprises functions linguistic fields of phonetics, syntax, semantics and pragmatics, in order to attain language achievements. In the present study was an attempt to explore which specific function of working memory is activated in order to decode each linguistic field of analysis. Specifically, several studies upon phonological memory indicate that there was a correlation with the linguistic field of phonetics as in the field of phonetics it was observed that there was a relation between phonological loop of memory and phonological awareness. The experimental findings showed that factors such as non word repetition (NWR) and word length, have an impact of the phonological memory function. Consequently, several studies also demonstrated that short-term memory is activated in order to decode syntactic structures of language. It is also noted the effect of short-term memory in syntactic parsing and syntactic disambiguation. Proceeding in further examination of the relation between working memory and text comprehension, researchers draw their attention between semantic memory and semantics. Coherent causality among sentences, as well as hierarchical structure of the text and the organization of text information based on their importance, seem to have a main function in the activation of semantic memory in the process of text understanding. Furthermore, linguistic phenomena such as anaphoric reference and lexical ambiguity are also parameters the comprehension of those is influenced by semantic memory. Lastly, research have paid much attention on the connection between episodic buffer and pragmatics. Investigation illustrated that the experience of episodic events which enhance cognitive knowledge facilitate their representation in memory, especially when Both schema-typical and schemaatypical activities are involved to the episodic event. Finally, researchers mark also the fact that comprehensibility and recall are correlated to the amount of inherent plot structure in the story, independent of passage content. 
In the second part of this paper it is attempted the demonstration of several representative processes and ICT tools for assessment, intervention and training of memory disfunctions in relation to language comprehension. .According to this, researchers also focus on the investigation of interactive media and human computer interaction in order to enhance memory for text comprehension. It is also worth presenting the Automated Working Memory Assessment (AWMA), a tool that gives the chance to users to assess memory deficits as well as the Visual Patterns Test (VPT) which was designed to measure short-term visual memory largely independent of its spatiosequential component. Following, authors establish the function of Working Memory Rating Scale (WMRS), which correlates with behavioural difficulties of children with poor working memory and also, The Mental Attributes Profiling System (MAPS) battery that consists of eight major domains of language-independent tests that have been frequently linked to reading development and/or disability. Finally, two more tools are demonstrated for assessment and memory training and these are Cognitive Profiling System (CoPs), a computerized psychometric assessment system that identifies children's cognitive abilities and APLo, an on-line system, for the assessment of children's oral speech and language abilities. As a conclusion, in the last years there has been a great progress in the research of the memory function in language comprehension but still there are more views on this filed that remain uninvestigated and undeniably, the impact of ICTs in this scientific field gives further prospects in the research of human cognitive abilities.

\section{$5 \quad$ References}

[1] https://en.wikipedia.org/wiki/Memory

[2] http://www.zeepedia.com/read.php?memory i functions of memory encoding and rec oding retrieval introduction to $p$ sychology $\& b=91 \& c=23$

[3] Susan E. Gathercole, Alan Baddeley,:"Evaluation of the role of phonological STM in the development of vocabulary in children. A longitudinal study", MRC Applied Psychology Unit, Cambridge, England, Journal of Memory and Language 28, 200-213 (1989) https://doi.org/10.1016/0749-596X(89)90044-2

[4] Erika Hoff, Cynthia Core, Kelly Bridges:"Non word repetition assesses phonological memory and is related to vocabulary development in 20 to 24 months olds",Journal Child Language 35, (2008), 903-916Cambridge University P ress

[5] Alan D. Baddeley, Neil Tomson and Mary Buchanan,:"Word length and the structure of STM" Medical Research Council, Applied Psychology Unit, University of Stirling Scotland. Journal of verbal learning and verbal behavior 14, 575-589 (1975) https://doi.org/10.1016/S0022-5371(75)80045-4

[6] Maryellen C. MacDonald, Marcel Adam Just, Patricia A. Carpenter:"Working Memory Constraints on the Processing of Syntactic Ambiguity" Cognitive Psychology 24, 56-98 (1992) https://doi.org/10.1016/0010-0285(92)90003-K

[7] Evelina Fedorenko, Edward Gibson, Douglas Rohde, :"The nature of working memory capacity in sentence comprehension: Evidence against domain-specific working memory resources", Journal of Memory and Language 54, $541-553$ (2009) https://doi.org/10.1016/ j.jml.2005.12.006 
Paper-Relation of Memory and Linguistic Fields and ICT Tools for Memory and Language Comprehen...

[8] Richard L. Lewis, Shravan Vasishth, Julie Van Dyke: "Computational principles of working memory in sentence comprehension", Trends in Cognitive Sciences Vol 10, No 10 https://doi.org/10.1016/j.tics.2006.08.007

[9] John B. Black and Hyman Bern: "Causal coherence and memory for events and narratives", Journal of verbal learning and verbal behavior 20, 267-275 (1981) https://doi.org/10.1016/S0022-5371(81)90417-5

[10] Gail McKoon: "Organization of information in text memory, Journal of verbal learning and verbal behavior 16, 247-260 (1977) https://doi.org/10.1016/S0022-5371(77)80050-9

[11] Maria N.K. Schwarz, August Flammer, "Text structure and title. Effects on comprehension and Recall" Journal of verbal learning and verbal behavior 20, 61-66 (1981) https://doi.org/10.1016/S0022-5371(81)90301-7

[12] Gail McKoon, Roger Ratcliff, Dartmouth College: "The comprehension processes and memory structures involves in anaphoric reference", Journal of verbal learning and verbal behavior 19, 668-682 ( 1980) https://doi.org/10.1016/S0022-5371(80)90355-2

[13] Akira Miyake, Marcel Adam Just, Patricia A. Carpenter: "Working Memory constraints on the resolution of lexical ambiguity. Maintaining multiple interpretations in neutral contexts" Journal of Memory and Language 33, 175-202 (1994) https://doi.org/10.1006/ jmla.1994.1009

[14] John B Black,Gordon H. Bower: "Episodes as chunks in Narrative Memory" Journal of verbal learning and verbal behavior 18, $309-318$ (1979) https://doi.org/10.1016/S0022$\underline{\text { 5371(79)90173-7 }}$

[15] Valerie Abbott, John B. Black, Edward E. Smith, Bolt: "The representation of scripts in memory", Beranek and Newman Inc. Journal of Memory and Language 24, 179-199 (1985) https://doi.org/10.1016/0749-596X(85)90023-3

[16] Perry W Thorndyke,: "Cognitive structures in comprehension and memory of narrative discourse" Cognitive psychology 9, 77-110, (1977) https://doi.org/10.1016/0010$\underline{0285(77) 90005-6}$

[17] Mina C. Johnson Glenberg: "Web-based training of meta-cognitive strategies for text comprehension: Focus on poor comprehenders", Reading and Writing December 2005, Volume 18, Issue 7, pp 755-786 https://doi.org/10.1007/s11145-005-0956-5

[18] Jerker Rönnberg, Mary Rudner, Catharina Foo \& Thomas Lunner: "Cognition counts: A working memory system for ease of language understanding (ELU)" pages S99-S105, International Journal of Audiology, Volume 47, Supplement 2, 2008

[19] Tracy P. Alloway, Susan E. Gathercole, Hannah Kirkwood \&Julian Elliott: "Evaluating the validity of the Automated Working Memory Assessment", Educational Psychology: An International Journal of Experimental Educational Psychology, Volume 28, Issue 7, 2008

[20] Janet Marie Wasowicz, Art Carl Maerlender :"Diagnostic system and method for phonological awareness, phonological processing, and reading skill testing 9 Oct 2001

[21] Sergio Della Sala, Colin Gray, Alan Baddeley, Nadia Allamano, Lindsey Wilsond: "Pattern span: a tool for unwelding visuo-spatial memory" Neuropsychologia Volume 37, Issue 10, September 1999, Pages 1189-1199 https://doi.org/10.1016/S0028-3932(98)00159$\underline{6}$

[22] Athanasios Drigas, , Georgia Kokkalia, Miltiadis D. Lytras : "ICT and collaborative colearning in preschool children who face memory difficulties" Computers in Human Behavior Volume 51, Part B, October 2015, Pages 645-651,Computing for Human Learning, Behaviour and Collaboration in the Social and Mobile Networks Era

[23] Tracy Packiam Alloway, Susan Elizabeth Gathercole, Hannah Kirkwood, Julian Elliott: "The working memory rating scale: A classroom-based behavioral assessment of working 
Paper-Relation of Memory and Linguistic Fields and ICT Tools for Memory and Language Comprehen..

memory Learning and Individual Differences, Volume 19, Issue 2, June 2009, Pages 242245 https://doi.org/10.1016/j.lindif.2008.10.003

[24] Elena Aristodemou, Tatjana Taraszow, Yiannis Laouris, Timotheos Papadopoulos and Pantelis Makris: "Prediction of Reading Performance Using the MAPS (Mental Attributes Profiling System) Multimodal Interactive ICT Application" Cyprus Neuroscience and Technology Institute, Nicosia, Cyprus, Proceedings of the 7th European Conference on eLearning

[25] Georgia K. Kokkalia and Athanasios S. Drigas: "Tools and E-tools for Memory and Attention Problems in Pre-school Education" NCSR DEMOKRITOS, Institute of Informatics and Telecommunications, Net Media Lab, Athens, Gr, iJES - Volume 3, Issue 3, 2015

\section{Authors}

Athanasios Drigas is a Research Director at IIT, N.C.S.R. Demokritos. He is the Coordinator of Telecoms Lab and founder of Net Media Lab since 1996. From 1990 to 1999 he was the Operational manager of the Greek Academic network. He has been the Coordinator of Several International Projects, in the fields of ICTs, and eservices (e-learning, e-psychology, e-government, einclusion, e-culture etc). He has published more than 270 articles, 7 books, 25 educational CD-ROMs and several patents. He has been a member of several International committees for the design and coordination of Network and ICT activities and of international conferences and journals. Also he has accepted several distinctions for his work (articles, projects, patents) (e-mail: dr@iit.demokritos.gr).

Alexandra Economou is an assistant professor at the University of Athens in the department of Psychology. She teaches undergraduate and postgraduate neuropsychology and experimental psychology courses at the University of Athens in the Department of Psychology while her interests focus on cognitive and biological psychology. Dr. Economou is also vice-president of the Greek Aphasia Association. (E-mail: aoikono@psych.uoa.gr)

Aglaia Tourimpampa is with N.C.S.R. 'Demokritos', Institute of Informatics and Telecommunications, Telecoms Lab - Net Media Lab, Agia Paraskevi, 153 10, Athens, Greece (e-mail: lidatou@gmail.com).

Article submitted 15 September 2016. Published as resubmitted by the authors 20 December 2016 